\title{
Virtual Pneumoperitoneum for Generating Virtual Laparoscopic Views Based on Volumetric Deformation
}

\author{
Takayuki Kitasaka $^{1}$, Kensaku Mori ${ }^{1}$, Yuichiro Hayashi ${ }^{1}$, Yasuhito Suenaga ${ }^{1}$, \\ Makoto Hashizume ${ }^{2}$, and Jun-ichiro Toriwaki ${ }^{3}$ \\ 1 Graduate School of Information Science, Nagoya University, \\ Furo-cho, Chikusa-ku, Nagoya, Aichi, 464-8603, Japan \\ \{kitasaka, kensaku, suenaga\}@is.nagoya-u.ac.jp \\ 2 Center for the Integration of Advanced Medicine and Innovative Technology, \\ Kyushu University Hospital, Fukuoka, 812-8582 Japan \\ 3 School of Computer and Cognitive Sciences, Chukyo University \\ 101 Tokodachi, Kaizu-cho, Toyota, Aichi, 470-0393, Japan \\ jtoriwak@sccs. chukyo-u.ac.jp
}

\begin{abstract}
This paper describes a method for generating virtual pneumoperitoneum based on volumetric deformation and its application to virtual laparoscopy. Laparoscopic surgery is now widely performed as a minimum-invasive surgery. Because a laparoscope has a very narrow viewing area, this limits the surgeon's viewable area. Making views that the abdominal wall is virtually elevated (virtual pneumoperitoneum) will be very helpful for intra-operative surgical navigation or pre-operative surgical planning. We deform original 3-D abdominal CT images so that the abdominal wall is virtually elevated. The entire process consists of five major steps: (a) extracting the abdominal wall, (b) elastic modeling, (c) elastic deformation of the model, (d) deformation of the original image, and (e) rendering virtual laparoscopic images. Virtual laparoscopic images are then generated from the deformed image. We have applied the method to three cases of 3-D abdominal CT images. From the experimental results, we confirmed that the abdominal wall was appropriately elevated by the proposed method. Laparoscopic views were very helpful for intra-operative surgical navigation as additional views of a surgeon or pre-operative surgical planning.
\end{abstract}

\section{Introduction}

Laparoscopic surgery has been widely performed as one of minimum-invasive surgery methods. Laparoscopic surgery is performed by making a working space (pneumoperitoneum) by infusing $\mathrm{CO}_{2}$ gas through a needle inserted to the peritoneum and inserting a laparoscope and forceps inside the abdominal cavity. Holes of $1 \mathrm{~cm}$ diameter are created on the abdominal wall for inserting such equipments. A surgeon performs surgery by watching a TV monitor that displays video images taken by the laparoscope camera. Although laparoscopic 
surgery does not require to widely open the abdomen and is less invasive, a laparoscope has very narrow viewing area. This limits the surgeon's viewable area. Sometimes it is very difficult for a surgeon to understand the positional relations between abdominal organs and lesions. This significantly increases the surgeon's load of laparoscopic surgery. Surgical planning and intraoperative surgical aid using three-dimensional (3-D) CT or MR images are very important.

Since surgical simulation or surgical planning using preoperative 3 -D images are quite useful, there are many researches on these topics[1234]. We have also developed a virtual endoscopy system that can be used for preoperative surgical planning and intraoperative surgical guidance [6]. Virtual endoscopy system is now widely used in the clinical field 5. If we could use a virtual endoscopy system and provide a surgeon virtual laparoscopic images rendered at arbitrary viewpoints and view directions during surgery, it would be very helpful for intraoperative surgical navigation or pre-operative surgical planning. A surgeon can make a surgical plan by checking the insertion points of a laparoscope and forceps by observing virtual laparoscopic views. Also it is possible to observe the positional relations between organs and lesions on virtual laparoscopic views. There are, however, very few reports for creating virtual laparoscopic views that simulates laparoscopy.

The virtual pneumoperitoneum requires to lift the abdominal wall for generating virtual laparoscopic images, since CT images are taken before pneumoperitoneum process in most cases. This paper proposes a method for elevating an abdominal wall based on volumetric deformation. We deform original 3-D abdominal CT images so that the abdominal wall is virtually elevated. Then, virtual laparoscopic images are generated from the deformed image. As far as we know, there is no report on simulating pneumoperitoneum for creating virtual laparoscopic images.

In Section 2 we describes the detail procedures for creating virtual laparoscopic views. Section 3 shows experimental result of virtual pneumoperitoneum. We add brief discussion in Section 4 .

\section{Method}

\section{$2.1 \quad$ Overview}

The entire process consists of five major steps: (a) extraction of abdominal wall, (b) elastic modeling, (c) elastic deformation of the model, (d) deformation of the original image, and (e) rendering of virtual laparoscopic images. We explain each steps below.

\subsection{Abdominal Wall Extraction}

We extract abdominal wall regions from input 3-D images. Abdominal wall regions comprise fat, muscle, and skin regions. Since these regions show very similar CT values, it is very difficult to segment abdominal wall regions based on simple thresholding. Therefore, we manually trace abdominal wall regions on slice 


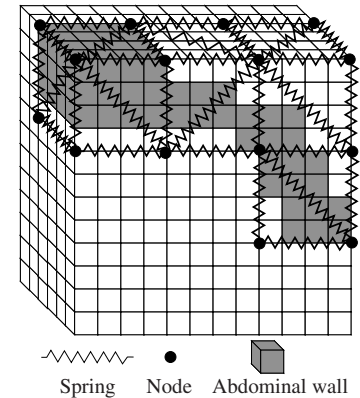

Fig. 1. Illustration of elastic cube allocation. Gray voxels are voxels forming the abdominal wall regions traced manually, while white voxels are non-abdominal wall regions that will not be deformed in the virtual pneumoperitoneum procedure. In this figure, we allocated one elastic cube for $4 \times 4 \times 4$ voxels regions for avoiding the figure becomes complex for understanding.

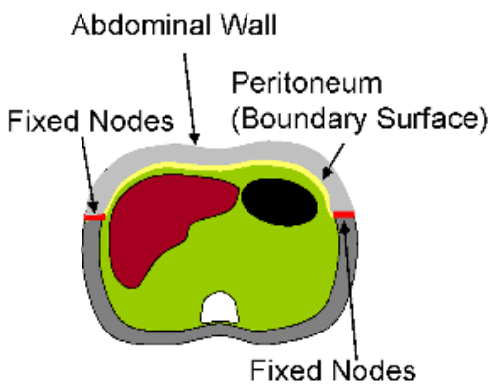

Fig. 2. An explanation of boundary surface and fixed nodes. thick yellow line shows peritoneum and red lines show the boundaries where nodes are fixed. The nodes on the dark gray boundaries are not forced to be moved during the deformation process.

images. The traced regions are used as the target areas of virtual pneumoperitoneum.

\subsection{Elastic Modeling}

Tetrahedron Division. In the proposed method we deform only abdominal wall regions for simplification, although underlying organs such as the liver, the colon, or the kidney is also deformed during pneumoperitoneum. In the elastic modeling process, we model the abdominal regions extracted in the above step by using a node and spring model. The node and spring model can compute deformation of the target regions by calculating forces working on each node and then moving the nodes to the directions of the resultant force.

We divide the extracted abdominal region into a set of cubes for reducing the computational time of deformation. Each cube consists of $8 \times 8 \times 8$ voxels. Figure 1] shows an example of elastic cube allocation. We consider vertices of a cube as nodes and assign springs on the edges and the diagonals on the surfaces of a cube (Fig. 11). One cube is divided into five tetrahedrons by this tessellation. Each tetrahedron consists of four nodes and six springs. The natural length of a spring is the distance between two connected nodes. We fix the nodes on the boundary surfaces that exist between the extracted abdominal walls and other regions as shown in Fig. 2

Forces for deformation. We deform the abdominal wall by adding virtual air pressure to the boundary surfaces. The abdominal lifting is achieved by calculating elastic deformation of tetrahedrons. In this paper, we consider three 
types of forces working on nodes in the deformation steps: (a) force caused by springs $\mathbf{F}_{s}$, (b) force that maintains the volumes of tetrahedrons $\mathbf{F}_{v}$, and (c) forces caused by air pressure $\mathbf{F}_{b}$. The type (c) force is considered only for the nodes on the boundary surfaces. Here, we denote a set of nodes that are connected to the $i$-th node by springs as $N_{i}$. A set of tetrahedrons that has the $i$-th node as their vertices is denoted as $T_{i}$.

In the $n$-th iteration step of deformation calculation, the force $\mathbf{F}_{i}(n)$ working on the $i$-th node is calculated as the resultant force of: (a) the force caused by the springs between the $i$-th node and the nodes connected to the $i$-th node and (force by springs) (b) the force caused by tetrahedrons that have the $i$-th node (force for preserving volume and force by air pressure). Hence the resultant force $\mathbf{F}(n)_{i}$ is described as

$$
\mathbf{F}_{i}(n)=\sum_{j \in N_{i}} \mathbf{F}_{s_{i, j}}(n)+\sum_{t \in T_{i}} \mathbf{F}_{v_{i, t}}(n)+\mathbf{F}_{b_{i}},
$$

where $\mathbf{F}_{s_{i j}}$ means the force caused by the spring between the $i$-th node and the $j$-th node connected to it, $\mathbf{F}_{v_{i t}}$ the force caused by the $t$-th tetrahedron that has the $i$-th node as its vertex.

\subsection{Iterative Deformation}

Each node is moved to the direction of the force working on it at very small distance. Moving distance is in proportion to the magnitude of the calculated force. We iteratively calculate the movement of each node. At the $n$-th iteration step, the position of the $i$-th node for the $(n+1)$-th iteration step $\mathbf{R}_{i}(n+1)$ is computed by

$$
\mathbf{R}_{i}(n+1)=\mathbf{R}_{i}(n)+\Delta s \mathbf{F}_{i}(n),
$$

where $\Delta s$ is a constant that controls the distance of movement at each iteration step. The movements of nodes are sequentially performed along the ordered list that holds the nodes. We iterate this movement step until

$$
\sum_{i \in S}\left\|\mathbf{R}_{i}(n+1)-\mathbf{R}_{i}(n)\right\|<\beta,
$$

where $\beta$ is a constant for determining convergence of the movement.

\subsection{Reconstruction of Volumetric Image}

After the deformation process, we reconstruct a volumetric image from the original image by using the relation between tetrahedrons of before- and afterdeformation. This process generates a deformed volumetric image where the abdominal wall is elevated.

There is one-to-one relation between vertices of a tetrahedron of after- and before-deformation. We deform the original 3-D image by using this relations. 


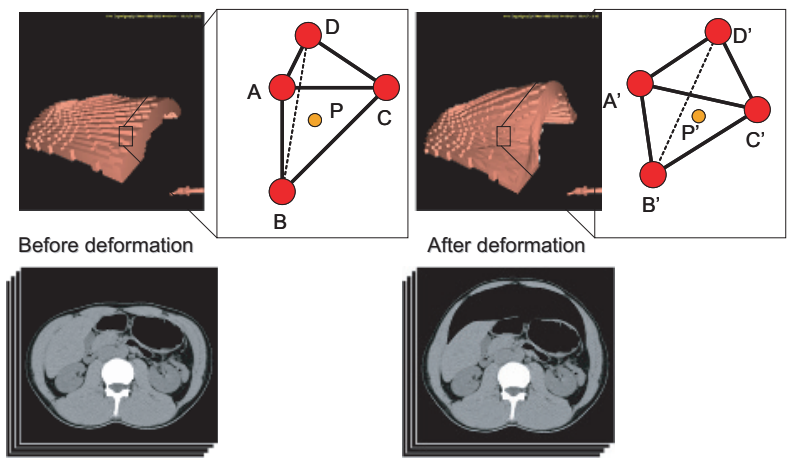

Fig. 3. Geometric correspondence for volumetric image reconstruction. Points A, B, $\mathbf{C}$, and $\mathbf{D}$ of before-deformation tetrahedron correspond to points $\mathbf{A}^{\prime}, \mathbf{B}^{\prime}, \mathbf{C}^{\prime}$, and $\mathbf{D}^{\prime}$ of deformed tetrahedron. Point $\mathbf{P}$, which is inside after-deformation tetrahedron, corresponds to point $\mathbf{P}$ inside before-deformation tetrahedron.

Let us consider the tetrahedron $t$ and its deformed one $t^{\prime}$. We denote vertices of a tetrahedron $t$ as $\mathbf{A}, \mathbf{B}, \mathbf{C}$, and $\mathbf{D}$. The vertices of the deformed tetrahedron $t^{\prime}$ are also described as $\mathbf{A}^{\prime}, \mathbf{B}^{\prime}, \mathbf{C}^{\prime}$, and $\mathbf{D}^{\prime}$ (Fig. 3 A point $\mathbf{P}^{\prime}$, which is inside of the deformed tetrahedron $t^{\prime}$, is represented by using four positional vectors $\mathbf{A}^{\prime}$, $\mathbf{B}^{\prime}, \mathbf{C}^{\prime}$, and $\mathbf{D}^{\prime}$ and four real variables $u, v, w$, and $x$ as

$$
\mathbf{P}^{\prime}=u \mathbf{A}^{\prime}+v \mathbf{B}^{\prime}+w \mathbf{C}^{\prime}+x \mathbf{D}^{\prime},
$$

where $u, v, w$, and $x$ satisfy

$$
\begin{array}{r}
u+v+w+x=1, \\
0 \leq u, v, w, x \leq 1 .
\end{array}
$$

By using Eqs. (4), (5), and (6), we can obtain actual values of $u, v, w$, and $x$. The point $\mathbf{P}$ of the tetrahedron $t^{\prime}$, which corresponds to $\mathbf{P}^{\prime}$, is also represented by using $u, v, w$, and $x$ as

$$
\mathbf{P}=u \mathbf{A}+v \mathbf{B}+w \mathbf{C}+x \mathbf{D} .
$$

The CT value on the point $\mathbf{P}^{\prime}$ is calculated from the $\mathrm{CT}$ value on $\mathbf{P}$ of the original image by the tri-linear interpolation. Consequently, we obtain the deformed $\mathrm{CT}$ image by calculating $\mathrm{CT}$ values on all grid points of the image.

\subsection{Visualization}

The deformed image is visualized by volume rendering method [7 for obtaining virtual laparoscopic image. It is possible to generate virtual laparoscopic view by putting a viewpoint of the virtual camera inside the virtually generated abdominal cavity. We can also color the liver, the kidney, and the descending aorta in virtual laparoscopic views by using the segmentation results of the organs. 


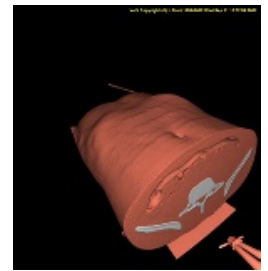

1000

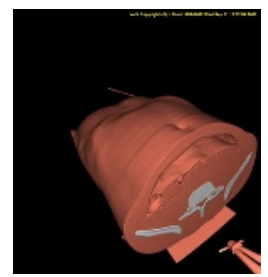

9000

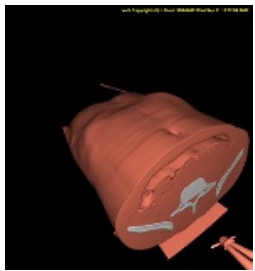

3000

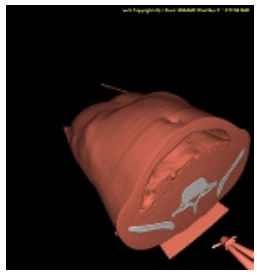

11000

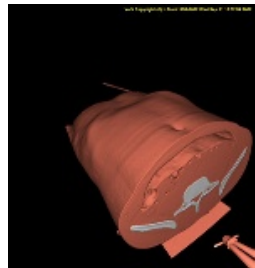

5000

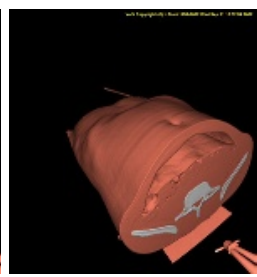

13000

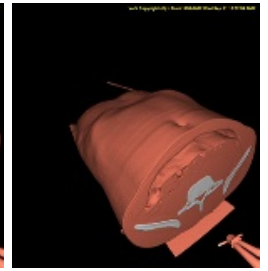

7000

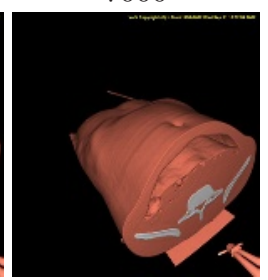

15000

Fig. 4. Progress of abdominal wall elevation. Numbers of iterations in elastic deformation are shown below each figure. These figures show the abdominal wall is gradually elevated by our method.

\section{Experiments}

We have applied the proposed method to three cases of 3-D abdominal CT images and generated virtual laparoscopic images. Acquisition parameters of the CT images are: $512 \times 512$ pixels, 0.625 (Cases A and B) or 0.606 (Case C) mm / pixel, 489 (Case A), 171 (Case B), 229 (Case C) slices, 2.0 mm slice thickness, and $1.0 \mathrm{~mm}$ reconstruction interval. The parameter used in the experiments is adjusted experimentally so that the proposed method can generate appropriate virtual pneumoperitoneum images. Actual value of the parameter is set to $\Delta s=$ 0.3. The abdominal wall regions are traced by one of the authors (Y. Hayashi).

The results of virtual pneumoperitoneum are shown in Fig. 4. This figure shows the progress of virtual pneumoperitoneum. It is clear that the abdominal wall is gradually lifted by the proposed method. An example of the deformed CT slices is shown in Fig. 5. Abdominal cavity is generated just above the stomach and the liver. Figure 6 shows a visualization of the liver, the descending aorta, the kidney, and the spleen, which were segmented by the method described in the reference 8] in the virtual laparoscopic image. A semi-translucent display technique was used. Figure 7 also shows a virtual laparoscopic view generated from the contrasted CT images (Case B). The liver and stomach regions are clearly visualized on these images. It took 75 minutes for the deformation process by a conventional PC that equips Intel Pentium III $933 \mathrm{MHz}$ processor.

\section{Discussion}

As shown in Figs. 4.7] the proposed method can generate virtual laparoscopic views from abdominal CT images, which are taken before a real pneumoperi- 


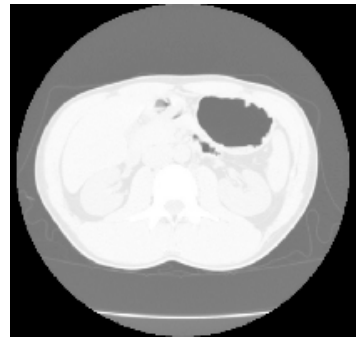

(a)

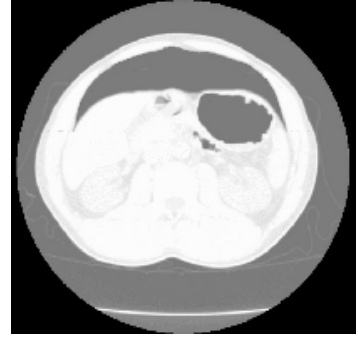

(b)

Fig. 5. Example of deformed slice images (Case A). (a) before deformation and (b) after deformation.

toneum process, by employing image deformation techniques based on the nodespring model. Virtual laparoscopic views depict the inside of the abdominal cavity and organs from arbitrary viewpoints view directions. Thus, a medical doctor can see the positional relation between lesions and the surrounding organs using these views before surgery. The virtual pneumoperitoneum proposed here can also be considered as a similation of pneumoperitoneum. A surgeon can intuitively determine the relation of each organ and where to insert instruments: a trocar, a laparoscope, and forceps. Therefore, virtual laparoscopic views generated by our method play an important role as images for surgical planning or as intraoperative reference images of laparoscopic surgery. We intend to use the generated virtual laparoscopic views in this paper as the reference images for making surgical plans before actual surgery, for understanding the relation of each organ, and for auxiliary views of a real laparoscope. The generated images are not used for surgical navigation that requires half a millimeter of guidance accuracy. We believe the simple elastic model employed here is enough for these purposes.

The elastic model in this paper is constructed only for abdominal wall regions. However, if we elevate the abdominal wall by inflating the abdominal cavity, the organ existing under the peritoneum also deforms because of air pressure. We ignored the deformation of organs here. As the future work, we need to develop a method that can simulate the deformation of abdominal organs and reduce the computation time. In addition, we need to improve a method that can clearly visualize organs existing inside the abdominal cavity, though the visibility of the segmented organs is improved by coloring the organs as shown in Fig. 6

\section{Conclusion}

This paper proposed a method for virtual elevation of the abdominal wall for generating virtual laparoscopic views. Elastic modeling was used for simulating elevation. Virtual laparoscopic views were obtained from the image that is deformed by using the deformation results of the elastic model. Because CT images 


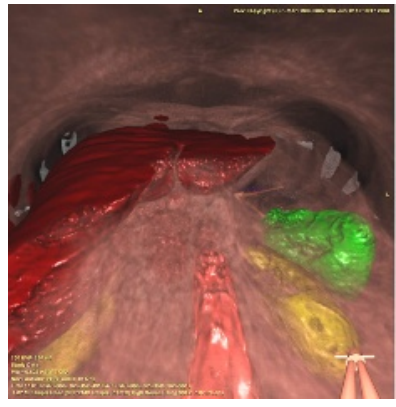

Fig. 6. Virtual laparoscopic view that visualizes the liver, the descending aorta, the kidney, and the spleen in the virtual laparoscopic image by employing semi-translucent display (Case A). The liver is colored in dark red, the kidney in yellow, the descending aorta in green, and the spleen in green.

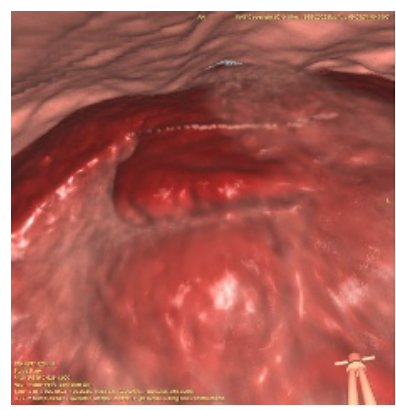

Fig. 7. Virtual laparoscopic view generated from contrasted CT images (Case B).

were taken before the elevation process, our method is quite useful for rendering virtual laparoscopic images from such images. Future work includes: (1) validation of the deformation results, (2) automated extraction of the abdominal wall, (3) application to many cases, and (4) reduction of the computation time.

Acknowledgments. The authors thank Dr. Shigeru Nawano of National Cancer Center East, Japan for providing CT images and foe his useful comments for the viewpoint of the medical field. The authors also thank our colleagues for their suggestions and advices. Parts of this research were supported by the Grant-In-Aid for Scientific Research from the Ministry of Education, the 21st century COE program, the Grant-In-Aid for Scientific Research from the Ministry of Education, Culture, Sports, Science, and Technology, Japan Society for Promotion of Science, and the Grant-In-Aid for Cancer Research from the Ministry of Health and Welfare.

\section{References}

1. N. Ayache, S. Cotin, H. Delingette et al., "Simulation of endoscopic surgery," Minimally Invasive therapy and Allied Technologies, vol. 7, no. 2, pp. 71-77, 1998

2. A. E. Kerdok, S. M. Cotin, M. P. Ottensmeyer et al., "Truth cube: Establishing physical standards for soft tissue simulation," Medical Image Analysis, vol. 6, no. 3, pp. 283-291, 2003

3. N. Suzuki and S. Suzuki, "Surgery simulation system with haptic sensation and modeling of elastic organ that reflect the patients' anatomy," Proc. of Surgery Simulation and Soft tissue Modeling, Lecture Notes in Computer Science, vol. 2673, pp. 155-164, 2003 
4. M. A. Audette, A. Fuchs, O. Astley et al., "Towards patient-specific anatomical model generation for finite element-based surgical simulation," Proc. of Surgery Simulation and Soft tissue Modeling, Lecture Notes in Computer Science, vol. 2673, pp. 340-351, 2003

5. P. Rogalla, J.Terwisscha van Scheltinga, B. Hamm eds., "Virtual Endoscopy and Related 3D Techniques," Springer, Berlin, 2001

6. K. Mori, A. Urano, J. Hasegawa et al., "Virtualized endoscope system -An application of virtual reality technology to diagnostic aid," IEICE Trans. on Information and System, vol. E79-D, no. 6, pp. 809-819, 1996

7. K. Mori, Y. Suenaga and J. Toriwaki, "Fast software-based volume rendering using multimedia instructions on PC platforms and its application to virtual endoscopy," Medical Imaging 2003: Physiology and Function: Methods, Systems, and Application, Proceedings of SPIE, vol. 5031, pp. 111-122, 2003

8. K. Yokoyama, T. Kitasaka, K. Mori et al., "Liver region extraction from 3D abdominal X-ray CT images using distribution features of abdominal organs," Journal of Computer Aided Diagnosis of Medical Images, vol. 7, no. 4-3, 2003 